\title{
3 Research Suare

\section{MiR-6838-5p enhances the growth and invasion of renal cell carcinoma dependent on DMTF1 via inhibiting the ARF-p53 pathway}

\section{Xiao-Qiang Zhai}

the Second Affiliated Hospital of Medical School, Xi'an Jiaotong University

\section{Yan Wu}

Xijing Hospital, Air Force Military Medical University

Dong Zhang

the Second Affiliated Hospital of Medical School, Xi'an Jiaotong University

He-Cheng Li

the Second Affiliated Hospital of Medical School, Xi'an Jiaotong University

Tie Chong

the Second Affiliated Hospital of Medical School, Xi'an Jiaotong University

Jun Zhao ( $\nabla$ zhaojun_xj@126.com )

the Second Affiliated Hospital of Medical School, Xi'an Jiaotong University https://orcid.org/0000-

0002-4646-8050

Primary research

Keywords: Renal cell carcinoma, miR-6838-5p, DMTF1, ARF-p53, RNA-RNA interaction

Posted Date: April 15th, 2020

DOI: https://doi.org/10.21203/rs.3.rs-22399/v1

License: (c) (1) This work is licensed under a Creative Commons Attribution 4.0 International License.

Read Full License 


\section{Abstract}

Backgroud: Renal cell carcinoma (RCC) is one of the most common renal malignancies in the urinary system. Numerous studies have demonstrated that miRNAs can regulate tumorigenesis and progression, while the underlying molecular mechanism for miR-6838-5p involved in RCC development is still largely unknown.

Methods: The relative expression of miR-6838-5p in RCC tissues, RCC cell lines, adjacent normal tissues and normal renal epithelial cells was detected by reverse transcription polymerase chain reaction (RTqPCR). In vitro studies, cell proliferation and invasion in human RCC cell lines ACHN and 786-0 were evaluated by CCK-8 assay, Transwell assay, Colony formation assay and Flow cytometry. Bioinformatics analysis, luciferase report analysis and Western blot assay were proved that Cyclin D binding myb-like transcription factor 1 (DMTF1) is the target of miR-6838-5p.

Results: Our study confirmed that miR-6838-5p was upregulated in RCC tissues $(30 / 42,77.43 \%, \mathrm{P}<0.01)$ and RCC cell lines $(P<0.05)$ compared to adjacent normal tissues and normal renal epithelial cells. In vitro studies demonstrated that overexpression of miR-6838-5p significantly increased cell proliferation and invasion in human RCC cell lines ACHN and 786-O $(P<0.05)$, whereas inhibition of miR-6838-5p inhibited cell proliferation and invasion $(P<0.05)$. Furthermore, we found that miR-6838-5p binds to the wild-type DMTF1 3'UTR. In addition, we found that DMTF1 was downregulation in RCC tissues and cell lines. Meanwhile, it was demonstrated in ACHN cells that overexpression of miR-6838-5p inhibited the expression of DMTF1. Finally, we also confirmed that the interaction of miR-6838-5p overexpression and DMTF1 overexpression might rescue the inhibitory effects of overexpression of miR-6838-5p on the expression of phosphatase and tensin homolog (PTEN), p53, Murine double minute 2 (MDM2) and alternative reading frame (ARF) in the DMTF1-mediated ARF-p53 downstream pathway.

Conclusions: Our research shows that miR-6838-5p enhances the growth and invasion of renal cell carcinoma dependent on DMTF1 via inhibiting the ARF-p53 pathway, which suggest that miR-6838-5p can be used as a marker for the diagnosis of RCC.

\section{Background}

Renal cell carcinoma (RCC) is the eighth most common cancer in the United States. It is estimated that 73,820 new cases will occur in 2019 , accounting for $4.2 \%$ of all cancers, of which 14,770 will be fatal, accounting for approximately $2.4 \%[1]$. RCC is the third most common malignancy in the female genitourinary system, and the second most common malignancy in men[2]. In 2016, WHO classified renal cell carcinoma into sixteen categories based on RCC predominant cytoplasmic features, architectural features, anatomic location of tumors, and correlation with a specific renal disease background, as well as molecular alterations pathognomonic for RCC subtypes, or familial predisposition syndromes[3]. In the early stages of RCC, patients have no characteristic clinical features, and about $30 \%$ of patients show signs of metastasis at diagnosis[4]. In addition, the prognosis of RCC patients remains poor $[5,6]$, so it is 
necessary to explore further potential mechanisms of RCC. Therefore, it is particularly important to identify novel biomarkers for diagnosis or delivery of RCC targeted therapy.

MicroRNAs (miRNAs), highly conserved small RNA molecules, are small fragments of 20-24 nucleotide non-coding RNA processed from pri-miRNA. MiRNAs are a type of regulator of post-transcriptional levels that inhibits the expression of these genes by binding to certain sequences of the 3'UTR of the target gene, thereby regulating the time of aging and cancer progression[7, 8]. MiRNAs have been extensively studied in renal cell carcinoma. Previous studies have demonstrated that MiR-23a-3p targeted binding Proline-Rich Nuclear Receptor Coactivator 2 (PNRC2) regulates the occurrence of RCC[9]. Both miR-200c$3 p$ and miR-96 have been shown to regulate the progression of RCC[10]. However, the regulation mechanism of miR-6838-5p in RCC progression is still unclear and remains to be studied.

Cyclin D binding myb-like transcription factor 1 (DMTF1), was initially isolated using cyclin D2 as bait in the yeast two-hybrid screening of the mouse T-lymphocyte library. Studies have found that spontaneous and oncogene-induced tumor formation can be accelerated in DMTF1 deletion and heterozygous mice[11, 12]. Previous studies have shown that DMTF1 appears as a key protein that links the rat sarcoma/rapidly accelerated fibrosarcoma/extracellular regulated protein kinases (Ras/Raf/ERK) growthpromoting signal to the alternative residing frame/protein 53/protein 21 (ARF/p53/p21) tumor suppressor network[13]. DMTF1 activates the tumor suppressor gene replacement reading frame protein $14^{\mathrm{ARF}}\left(\mathrm{p} 14^{\mathrm{ARF}}\right)$, and increased $\mathrm{p} 14^{\mathrm{ARF}}$ levels cause cell cycle arrest and cell death primarily in a $\mathrm{p53}-$ dependent manner. Wild-type ARF and p53 are typically retained in DMTF1 deletion and heterozygous mice, so it is understood that DMTF1 is a physiological regulator of the ARF-p53 pathway. In addition, studies have found that tumors can occur in mice without p53 mutations or ARF deletions, further illustrating the importance of DMTF1 in the p53 pathway[12]. In cancers such as bladder cancer[14], colorectal cancer[15] and breast cancer[16], it has been reported that overexpression or knockdown of DMTF1 can inhibit or promote cancer progression. In the present study, it was confirmed that miR-6838$5 p$ inhibits the expression of DMTF1 to promote proliferation and invasion of RCC cells by targeting DMTF1.

\section{Materials And Methods}

\section{Patient tissue samples}

This study was approved by the Ethics Committee of the Second Affiliated Hospital of Medical School, Xi'an Jiaotong University (Xi'an, China), which was conducted according to the Helsinki Declaration. The RCC tissue samples were collected from 42 RCC patients ( $45.0 \pm 15.3$ years) who experienced surgical resection but did not receive chemotherapy or radiation therapy. The corresponding adjacent normal tissues were also taken from the edge of the RCC tissues by $3 \sim 4 \mathrm{~cm}$ which were no obvious tumor cells. All tissues were frozen in liquid nitrogen and stored at $-80^{\circ} \mathrm{C}$ for subsequent testing.

\section{Cell lines and cell culture}


The human renal epithelial cell lines (HK-2 and 293T) and RCC cell lines (Caki-1, ACHN, 786-P and 786-0) utilized in the present study were purchased from the Institute of Cell Research, Chinese Academy of Sciences (Shanghai, China). The authenticity of cell lines has been verified by short tandem repeat analysis. All cells were cultured in Roswell Park Memorial Institute 1640 medium (RPMI-1640, Gibco, BRL, Carlsbad, CA, USA) and supplemented with of $10 \%$ fetal bovine serum (Sigma-Aldrich, St. Louis, MO, USA), then incubated in a $37^{\circ} \mathrm{C}$ incubator with a humidified atmosphere of $5 \% \mathrm{CO}_{2}$.

\section{Reverse transcription polymerase chain reaction (RT-qPCR)}

Total RNA was extracted from cells and tissues according to the instruction of TRIzol reagent (Invitrogen, Carlsbad, CA, USA) and purified with the RNeasy Maxi kit (Qiagen, Dusseldorf, Germany) according to the manufacturer's protocols. MiScript Reverse Transcription kit (Qiagen, Dusseldorf, Germany) was used to reverse transcription with $1 \mu \mathrm{g}$ total RNA of each sample and miScript SYBR ${ }^{\circledR}$ Green PCR kit (Qiagen, Dusseldorf, Germany) was used to detect the expression level of miR-6838-5p following the manufacturer's protocols. We used U6 or GADPH as endogenous control, respectively. PCR program in real-time PCR system (ABI7500, Applied Biosystems, Waltham, MA, USA), the detailed process was as follows: 1 minute at $95^{\circ} \mathrm{C}$ followed by 35 cycles of 20 seconds at $95^{\circ} \mathrm{C}$, then 10 seconds at $56^{\circ} \mathrm{C}$ and 15 $s$ at $72{ }^{\circ} \mathrm{C}$. Finally, the relative expression levels of miR-6838-5p were calculated using the $2^{-\Delta \Delta \mathrm{Ct}}$ method.

\section{Cell transfection}

The NC mimic, miR-6838-5p mimic, NC inhibitor, anti-miR-6838-5p, Scramble and DMTF1 siRNA, Vector were purchased from Invitrogen (Carlsbad, CA, USA). DMTF1 overexpressed plasmid (pcDNA3.1-DMTF1) was synthesized by Thermo Fisher Scientific (Waltham, MA, USA). Transfection and co-transfection of these oligonucleotides were carried out with Lipofectamine Plus reagent (Invitrogen, Carlsbad, CA, USA) according to the manufacturer's instructions.

\section{Western blotting}

We used RIPA lysis buffer (CW Biotech, Beijing, China) to lyse the cells to extract total protein and measure protein concentration by BCA protein assay kit (Thermo Fisher Scientific, Waltham, MA, USA). To separate protein samples in equal amounts, $10 \%$ SDS-PAGE must be used. Next, we transferred and blocked the complex onto a nitrocellulose membrane (General Electric Co, USA). After blocked with $5 \%$ skim milk, the blots were probed with primary antibodies. Following washed with PBS, the horseradish peroxidase-conjugated Goat Anti-Rabbit IgG secondary antibodies (ab205718, 1区2, 000) were added. Next, we incubated the membrane for 1 hour at room temperature. The primary antibodies used in this study including Rabbit Anti-DMTF1 antibody (ab246945, 1:500), Rabbit Anti-PTEN antibody (ab32199, 1:500) , Rabbit Anti-p53 antibody (ab32389, 1:100), Rabbit Anti-MDM2 antibody (ab38618, 1:400) and Rabbit Anti-ARF antibody (ab77581, 1:300) (Cell Signaling Technology, Inc. Danvers, MD, USA). We then uniformly added the chemiluminescence reagent to the film and developed the image with a developer. 
We used Tubulin as an endogenous control to normalize protein expression. The fold change versus control group was the demonstration of target proteins' relative expression levels.

\section{Cell viability assay}

The CCK-8 proliferation assay kit was used to determine the proliferation of cells. First, cells were incubated for 24 hours, then $3 \times 10^{3}$ cells were seeded into 96 -well plates and cells were transfected with the indicated RNA duplexes. Then, the cells were treated with $10 \mu \mathrm{L}$ of the cell counting solution (WST-8, Dojindo Laboratories, Tokyo) at 0, 24 and 72 hours, respectively, and incubated for an additional 2 hours. The absorbance (OD) value was measured at $450 \mathrm{~nm}$ using a microplate reader.

\section{Transwell invasion assay}

The invasive ability of ACHN and 786-0 cells was determined using an $8.0 \mu \mathrm{m}$ 24-well Boyden chamber. For the intrusion test, first, we pre-coated Matrigel in the chamber, then inoculated $2 \times 10^{5}$ cells in the serum-free medium into the upper layer of the chamber, and then added the intact medium as a chemotactic agent to the lower chamber. After 24 hours, we fixed the membrane with methanol and stained with $0.1 \%$ crystal violet, then selected five randomly selected fields of view $(\times 100)$ to count the invading cells.

\section{Colony formation assays}

For colony formation analysis, cells were seeded into 6-well plates at a concentration of 500 or 2000 cells/well, ensuring that the medium was changed every 5 days. After the cells were incubated for 7-14 days, cells were washed with PBS, fixed with $4 \%$ paraformaldehyde, and then stained with $0.5 \%$ crystal violet. Finally, the number of colony formations was calculated.

\section{Flow cytometry}

Cells were washed with cold PBS and fixed in cold $75 \%$ ethanol for 30 minutes at $4{ }^{\circ} \mathrm{C}$. The fixed cells were washed three times and treated with ribonuclease. The resulting cells were stained with propidium iodide $\left(50 \mu \mathrm{g} / \mathrm{mL}\right.$ ) overnight at $4^{\circ} \mathrm{C}$ (Abeam, Cambridge, UK) and then subjected to flow cytometry analysis at $488 \mathrm{~nm}$ challenge (BD Biosciences, Franklin Lakes, NJ, USA).

\section{Vector construction and luciferase assay}

The TargetScan Human database (http://www.targetscan.org/) was used to predict potential targets of miR-6838-5p. The 3'UTR of the DMTF1 sequence was amplified and cloned into a pGL4.10-report vector (Promega Corporation, Madison, WI, USA). Equal quantities of the pGL4.10-3'UTR-DMTF1 and Renilla expression vector pRL-TK (Promega Corporation) were co-transfected into the HEK293 cells. The luciferase activity was measured $48 \mathrm{~h}$ post-transfection using the Dual-Glo Luciferase Assay system (Promega Corporation). 


\section{Statistical analysis}

Statistical analyses were performed on SPSS 19.0 (IBM SPSS, Armonk, NY, USA). All quantitative results were presented as the mean \pm standard deviation (SEM) of three independent experiments. The significance of the differences between two groups, unless for paired comparison which was noted specially, was conducted with a two-tailed Student's t-test. Analysis of variance (ANOVA) was used to compare the significance between multiple groups. The correlations between miR-6838-5p and clinical characteristics were analyzed by Chi-square test. $P$-values $<0.05$ were considered to be statistically significant.

\section{Results}

\section{MiR-6838-5p was upregulated in RCC tissues and cell lines}

In this experiment, the relative level of miR-6838-5p in 42 pair of RCC tissues and adjacent nontumorous tissues were examined by RT-qPCR. As shown in Fig. 1A, the results determined that miR-6838-5p was upregulated in $77.43 \%$ (30/42) of in 42 RCC cases. Compared with normal tissues, we have noticed that miR-6838-5p was upregulated in tumor tissues (Fig. 1B). In addition, we demonstrated that miR-6838-5p had high expression in RCC cell lines including Caki-1, ACHN, 786-O and 786-P (Fig. 1C). These results suggested that miR-6838-5p was an event in RCC and could be a diagnostic marker in RCC.

\section{Overexpression of miR-6838-5p promotes $A C H N$ and $786-0$ cell proliferation and invasion}

To detect the effect of miR-6838-5p overexpression on the proliferation and invasion of RCC cells, miR6838-5p mimics were transfected into human $\mathrm{ACHN}$ and 786-0 cells which used to induce overexpression of miR-6838-5p. The results showed that the relative level of miR-6838-5p was upregulated in ACHN and 786-0 cells transfected with miR-6838-5p mimic (Fig. 2A and 2D). Next, CCK-8 and Transwell assay were used to detect the effect of miR-6838-5p on cell proliferation and invasion. As shown in Fig. 2B and 2E, we found that $\mathrm{ACHN}$ and $786-0$ cells enhanced cell proliferation transfected with miR-6838-5p mimic compared with $\mathrm{NC}$ mimic. Furthermore, we measured the invasiveness of $\mathrm{ACHN}$ and 786-0 cells transfected with miR-6838-5p mimic and NC mimic for $72 \mathrm{~h}$. As shown in Fig. $2 \mathrm{C}$ and 2F. ACHN and 786$O$ cells increased the number of invasive cells after transfection with miR-6838-5p mimic compared with $\mathrm{NC}$ mimic. These results indicated that miR-6838-5p overexpression enhanced human ACHN and 786-0 cells proliferation and invasion.

\section{Overexpression or inhibition of miR-6838-5p prolongs or shortens the S phase of human ACHN and 786- 0 , and increases or inhibits colony formation}

In order to explore the effect of overexpression or inhibition of miR-6838-5p on cell cycle, we used flow cytometry to detect the number of ACHN cells in different periods. MiR-6838-5p inhibitor was transfected into human ACHN and 786-O RCC cells to inhibit miR-675-3p expression. The number of ACHN cells in the $S$ phase were extended or shortened after transfection with miR-6838-5p mimic or anti-miR-6838-5p 
compared with NC mimic or NC inhibitor (Fig. 3A, B, C and D). However, the change in the G2/M phase was opposite to the S phase (Fig. 3A, B, C and D). As shown in Fig. 3E and F, the relative level of miR6838-5p was decreased transfected with anti-miR-6838-5p compared with NC inhibitor. In addition, Colony formation assay was performed to the effect on ACHN cells while transfection with overexpression or inhibition of miR-6838-5p (Fig. 3G, H, I and J).

\section{MiR-6838-5p directly targets the 3'UTR of DMTF1}

To predict the target gene of miR-6838-5p, TargetScan (http://www.targetscan.org/) and ChIPbase (http://rna.sysu.edu.cn/chipbase/) were used to verify DMTF1 can be combined with miR-6838-5p target. DMTF1 is a pro-cancer protein in renal cell carcinoma but is closely related to the arf-p53 pathway, and significant inhibition is responsible for the progression of renal cell carcinoma. As shown in Fig. 4A, the target sequence of miR-6838-5p is shown in the 3'-UTR of the Wild type DMTF1.

In order to validate this targeting relationship, dual luciferase reporter assay was executed. The result showed that miR-6838-5p significantly decreased the luciferase activity of the wild type DMTF1 in the 3'UTR of DMTF1 in HEK293 cells compared with the mutant type DMTF1.

\section{Overexpression of miR-6838-5p inhibits the expression of DMTF1, while inhibition of miR-6838-5p promotes the expression of DMTF1}

To further explore the regulation of miR-6838-5p on DMTF1, ACHN cells were transfected by overexpression or inhibition of miR-6838-5p vector. As shown in Fig. $5 A$ and $B$, the relative level of DMTF1 was decreased in tumor tissues compared with normal tissues, which was detected by western blotting. And the mRNA level of DMTF1 was significantly reduced in four RCC cells including Caki-1, ACHN, 786-0 and 786-P but not in HK-2 and 293-T (Fig. 5C). Next, we found that overexpression or inhibition of miR6838-5p inhibited or enhanced the relative level of DMTF1 by western blotting (Fig. 5D and 5E). These results indicated that overexpression or inhibition of miR-6838-5p inhibited or enhanced the expression of DMTF1.

Overexpression of DMTF1 promotes expression of ARF, p53, PTEN and MDM2 proteins while knockdown of DMTF1 inhibits these proteins in the downstream pathway of ARF-p53

As shown in Fig. 6A and 6B, overexpression or inhibition of DMTF1 enhanced or inhibited the relative level of DMTF1, which was determined by Western blotting. Similarly, overexpression or inhibition of DMTF1 promoted or suppressed the relative level of PTEN, p53, MDM2 and ARF (Fig. 6C and 6D). We further explored the effect of overexpression or knockdown of DMTF1 on proliferation and invasion of ACHN cells by CCK-8 and Transwell assays. The results demonstrated that overexpression or inhibition of DMTF1 inhibited or enhanced ACHN cell proliferation and invasion (Fig. 6E and 6F).

Co-transfection of miR-6838-5p overexpression and DMTF1 overexpression vectors into ACHN cells rescues the inhibitory effect of overexpression of miR-6838-5p on the DMTF1-p53 pathway 
As shown in Fig.7A, transfection of ACHN cells with miR-6838-5p overexpression vector alone increased the mRNA level of miR-6838-5p, but co-transfection of ACHN cells with overexpressed miR-6838-5p and overexpressed DMTF1 vector inhibited miR-6838-5p expression. In contrast to Fig. 7A, miR-6838-5p overexpression inhibited DMTF1 expression, whereas interaction of miR-6838-5p overexpression and DMTF1 overexpression rescued the inhibitory effect of miR-6838-5p overexpression in Fig. 7B. As shown in Fig. 7C and 7D, Western blot was used to detect that overexpression of miR-6838-5p suppressed the expression of ARF, p53, MDM2 and PTEN proteins in the downstream pathway of DMTF1-mediated ARF$\mathrm{p} 53$, while interaction of miR-6838-5p overexpression and DMTF1 overexpression reversed miR-6838-5p overexpression from inhibiting ARF-p53 downstream pathway proteins. As shown in Fig. 7E and 7F, Cotransfection of overexpressing miR-6838-5p and overexpressing DMTF1 vector to ACHN cells inhibited the proliferation and invasion of miR-6838-5p overexpression which was detected by CCK-8 and Transwell assay for $72 \mathrm{~h}$, respectively.

\section{Discussion}

Emerging evidences have shown that miRNAs play important roles in the development and progression of RCC[17-21]. These miRNAs can act as tumor suppressor genes or oncogenes by modulating the target genes. Therefore, the identification of specific miRNAs and their target genes in RCC contributes to understanding the occurrence and progression of RCC. However, so far, there are few studies on the expression and biological regulation mechanism of miRNA-6838-5p in RCC. In this study, we explored the cellular role and mechanism of miR-6838-5p in RCC, revealed the regulatory mechanism of miR-6838-5p on DMTF1 expression, and blocked miR-6838-5p as a RCC tumor carcinogen by modulating DMTF1. At present, there are few reports on the regulation of cancer by miR-6838-5p, and this experiment confirmed that miR-6838-5p has a cancer-promoting effect in RCC. Similar to miR-221, miR-21, miR-30b, miR-15a-5p, etc[22-24], miR-6838-5p also promoted the proliferation and invasion of RCC cells. Our results also showed that miR-6838-5p promoted the prolongation of S phase of ACHN and 786-0 cells and induced the formation of RCC cell colonies. The above results confirmed that miR-6838-5p has a cancerpromoting effect in RCC. When we treated ACHN cells with miR-6838-5p inhibitor, it was found that the pro-carcinogenesis of miR-6838-5p was significantly inhibited, indicating that miR-6838-5p does play a role in ACHN cells. Next, we predicted by software that miR-6838-5p can target the 3'UTR of DMTF1, and then confirmed that miR-6838-5p targets DMTF1 by regulating DMTF1, ARF, MDM2, p53 and PTEN in the DMTF1-P53 pathway. The expression of the protein plays a role in tumor suppression or cancer promotion. All above results indicate that miR-6838-5p may be used as a diagnostic marker for clinical RCC, with RCC and prognostic diagnosis.

Numerous studies have shown that DMTF1 controls the activity of the ARF-p53 tumor suppressor pathway by binding to the ARF promoter. It has been reported that human genes have three different splice variants (differential splicing), namely DMTF1a, DMTF1 $\beta$ and DMTF1y, whereas only DMTF1a activates the ARF promoter[25]. DMTF1 $\beta$ antagonizes the regulation of $p 14^{A R F}$ tumor suppressor by DMTF1 $\alpha$ and promotes cell proliferation. However, DMTF1 $\beta$ caused DMP1 $\alpha$-induced transactivation of 
the ARF promoter in a dose-dependent manner. In the cisplatin sensitivity test of breast cancer cells, it was found that DMTF1 transactivates the tumor suppressor gene alternate reading frame ( $\left.\mathrm{p} 14^{\mathrm{ARF}}\right)$. Elevated levels of p14 ${ }^{\mathrm{ARF}}$ cause cell cycle arrest and cell death in a p53-dependent manner[14]. ARF is an alternative reading frame product of the INK4a/ARF locus and is inactivated in many human cancers. Furthermore, ARF is a key regulator of cellular senescence, an irreversible cell growth arrest that inhibits tumor cell growth. It activates p53 by isolating MDM2, a p53 E3 ligase, in the nucleolus. As a tumor suppressor, ARF is closely related to p53 stability, which induces cell senescence and prevents tumor cell growth[12]. ARF releases p53 from MDM2, a well-known E3-ubiquitin ligase of p53, and captures MDM2 in the nucleolus by direct interaction, physically separating p53 and MDM2[26-28]. In this experiment, we infected ACHN cells with a vector that overexpresses or silences DMTF1 which found that it promoted or inhibited the expression of DMTF1, ARF, MDM2, p53 and PTEN in DMTF1-p53 signaling pathway, confirming that DMTF1 is DMTF1- Regulation of the p53 signaling pathway. Secondly, we co-transfected AMR cells with miR-6838-5p and overexpressed DMTF1 and found that the inhibition of miR-6838-5p on RCC cells was inhibited by inhibiting the expression of the above proteins in the ARF-p53 signaling pathway which clarifies the regulatory mechanism of miR-6838-5p in RCC.

\section{Conclusion}

These findings demonstrated that miR-6838-5p may be a new target of RCC treatment. Its biological mechanism is to promote the proliferation and invasion of RCC cells via targeting DMTF1 protein to inhibit ARF-PTEN pathway.

\section{Abbreviations}

RCC: Renal cell carcinoma; RT-qPCR: reverse transcription polymerase chain reaction; DMTF1هCyclin D binding myb-like transcription factor $1 \triangle \mathrm{PTEN} \rrbracket$ phosphatase and tensin homolog $\triangle \mathrm{MDM}$ 2खMurine double minute $2 \triangle A R F \bigotimes$ alternative reading frame; miRNAs: microRNAs; PNRC2: Proline-Rich Nuclear Receptor Coactivator 2; Ras: rat sarcoma; Raf: rapidly accelerated fibrosarcoma; ERK: extracellular regulated protein kinases; p53囚protein 53囚p21凶protein 21.

\section{Declarations}

\section{Acknowledgements}

l'd like to express my sincere thanks to all those who have lent me hands in the course of my writing this paper.

\section{Authors' contributions}

JZ conceived and designed the experiments. $X Z$ and $Y W$ performed the experiments. $X Z, Y W, D Z, H L, T C$ and $\mathrm{JZ}$ analyzed the data. $\mathrm{XZ}$ and $\mathrm{YW}$ wrote the manuscript. All authors read and approved the 
manuscript and agree to be accountable for all aspects of the research in ensuring that the accuracy or integrity of any part of the work are appropriately investigated and resolved.

\section{Funding}

None

\section{Availability of data and materials}

The datasets used and/or analyzed during the present study are available from the corresponding author on reasonable request.

\section{Ethics approval and consent to participate}

Patients' written informed consent was obtained and was approved by the Ethics Committee of the Second Affiliated Hospital of Medical School, Xi'an Jiaotong University (Xi'an, China).

\section{Consent for publication}

All authors agreed on the manuscript.

\section{Competing interests}

The authors declare that there is no conflict of interest regarding the publication of this paper.

\section{Author details}

1 Department of Urology, The Second Affiliated Hospital of Medical School, Xi'an Jiaotong University, Xi'an, Shaanxi 710004, China.

2 Department of Obstetrics and Gynecology, Xijing Hospital, Air Force Military Medical University, Xi'an, Shaanxi 710032, China.

\section{References}

1. Siegel RL, Miller KD, Jemal A.Cancer statistics. 2019. CA Cancer J Clin 2019; 69(1):7-34.

2. Miller KD, Nogueira L, Mariotto AB, Rowland JH, Yabroff KR, Alfano CM, Jemal A, Kramer JL. Siegel RL.Cancer treatment and survivorship statistics, 2019. CA Cancer J Clin. 2019;69(5):363-85.

3. Humphrey PA, Moch H, Cubilla AL, Ulbright TM, Reuter VE. The 2016 WHO Classification of Tumours of the Urinary System and Male Genital Organs-Part B: Prostate and Bladder Tumours. Eur Urol. 2016;70(1):120-3.

4. Alt AL, Boorjian SA, Lohse CM, Costello BA, Leibovich BC, Blute ML. Survival after complete surgical resection of multiple metastases from renal cell carcinoma. Cancer. 2011;117(13):2873-82. 
5. Zhang HM, Yang F-q, Chen S-J, Che J, Zheng J. -h.Upregulation of long non-coding RNA MALAT1 correlates with tumor progression and poor prognosis in clear cell renal cell carcinoma. Tumor Biol. 2015;36(4):2947-55.

6. Bianchi M, Sun M, Jeldres C, Shariat SF, Trinh Q-D, Briganti A, Tian Z, Schmitges J, Graefen M. Perrotte P.Distribution of metastatic sites in renal cell carcinoma: a population-based analysis. Ann Oncol. 2012;23(4):973-80.

7. Yusuf T. Editorial (Thematic Issue: "miRNA and Cancer; Computational and Experimental Approaches"). Curr Pharm Biotechno. 2014;15(5):429-9.

8. Qadir MI, Faheem AmiRNA. A Diagnostic and Therapeutic Tool for Pancreatic Cancer. Crit Rev Eukar Gene. 2017;27(3):197-204.

9. Quan J, Pan X, Li Y, Hu Y, Tao L, Li Z, Zhao L, Wang J, Li H, Lai Y, et al. MiR-23a-3p acts as an oncogene and potential prognostic biomarker by targeting PNRC2 in RCC. Biomed Pharmacother. 2019;110:656-66.

10. Li S, Feng Z, Zhang X, Lan D, Wu Y. Up-regulation of microRNA-200c-3p inhibits invasion and migration of renal cell carcinoma cells via the SOX2-dependent Wnt/beta-catenin signaling pathway. Cancer Cell Int. 2019;19:231.

11. Inoue K, Fry EA. Aberrant splicing of the DMP1-ARF-MDM2-p53 pathway in cancer. Int J Cancer. 2016;139(1):33-41.

12. Inoue K, Wen R, Rehg JE, Adachi M, Sherr CJ. Disruption of the ARF transcriptional activator DMP1 facilitates cell immortalization, Ras transformation, and tumorigenesis. Genes Dev. 2000;14(14):1797-809.

13. Inoue K, Fry EA. Tumor suppression by the EGR1, DMP1, ARF, p53, and PTEN Network. Cancer Invest. 2018;36:1-17.

14. Peng Y, Dong W, Lin TX, Zhong GZ, Huang J. MicroRNA-155 promotes bladder cancer growth by repressing the tumor suppressor DMTF1. Oncotarget. 2015;6(18):16043-58.

15. Yang X, Lou Y, Wang M, Liu C, Liu Y, Huang W. miR675 promotes colorectal cancer cell growth dependent on tumor suppressor DMTF1. Mol Med Rep. 2019;19(3):1481-90.

16. Niklaus NJ, Humbert M, Tschan MP. Cisplatin sensitivity in breast cancer cells is associated with particular DMTF1 splice variant expression. Biochem Biophys Res Commun. 2018;503(4):2800-6.

17. Luo C, Jiangnan Q. miR-181a Inhibits Cervical Cancer Development via Downregulating GRP78. Oncol Res. 2017;25(8):1341-8.

18. Lou N, Ruan A-M, Qiu B, Bao L, Xu Y-C, Zhao Y, Sun R-L, Zhang S-T, Xu G-H, Ruan H-L.miR-144-3p as a novel plasma diagnostic biomarker for clear cell renal cell carcinoma. Urol Oncol 2017; 35:36.e3736.e14.

19. Wang Y, Dong D, Jiang S, Zhang E, Zheng W, Mao L, Li W, Zhou J, Fan L, Cheng R, et al. miR-216b Post-Transcriptionally Downregulates Oncogene KRAS and Inhibits Cell Proliferation and Invasion in Clear Cell Renal Cell Carcinoma. Cell Physiol Biochem. 2018;49(5):1755-65. 
20. Liu L, Li Y, Liu S, Duan Q, Chen L, Wu T, Qian H, Yang S, Xin D. Downregulation of miR-193a-3p inhibits cell growth and migration in renal cell carcinoma by targeting PTEN. Tumour Biol. 2017;39(6):101042831771195.

21. Ishihara T, Seki N, Inoguchi S, Yoshino H, Tatarano S, Yamada $Y$, Itesako T, Goto Y, Nishikawa R, Nakagawa M. Expression of the Tumor Suppressive miRNA-23b/27b Cluster is a Good Prognostic Marker in Clear Cell Renal Cell Carcinoma. J Urol. 2014;192(6):1822-30.

22. Szabó Z, Szegedi K, Gombos K, Mahua C, Flaskó T, Harda K, Halmos G.Expression of miRNA-21 and miRNA-221 in clear cell renal cell carcinoma (ccRCC) and their possible role in the development of ccRCC. Urol Oncol-Semin Ori 2016:S1078143916301284.

23. Lu J, Yifan L, Tao H, Jia H, Jiaju L, Mingwei C, Min S, Zhimao J, Shangqi Y. Xiangming M.Identification of miR-30b as an oncogene in renal cell carcinoma. Mol Med Rep. 2017;15(4):183746.

24. Jin L, Li Y, He T, Hu J, Lai Y. MiR-15a-5p acts as an oncogene in renal cell carcinoma. Mol Med Rep. 2017;15(3):1379.

25. Tschan MP, Federzoni EA, Haimovici A, Britschgi C, Moser BA, Jin J, Reddy VA, Sheeter DA, Fischer $\mathrm{KM}$, Sun $\mathrm{P}$, et al. Human DMTF1 beta antagonizes DMTF1 alpha regulation of the p14(ARF) tumor suppressor and promotes cellular proliferation. Biochim Biophys Acta. 2015;1849(9):1198-208.

26. Carrasco-Garcia E, Moreno M, Moreno-Cugnon L, Matheu A. Increased Arf/p53 activity in stem cells, aging and cancer. Aging Cell. 2017;16(2):219-25.

27. Ko A, Han SY, Song J. Dynamics of ARF regulation that control senescence and cancer. BMB Rep. 2016;49(11):598-606.

28. Ko A, Han SY, Song J. Regulatory Network of ARF in Cancer Development. Mol Cells. 2018;41(5):381-9.

\section{Figures}




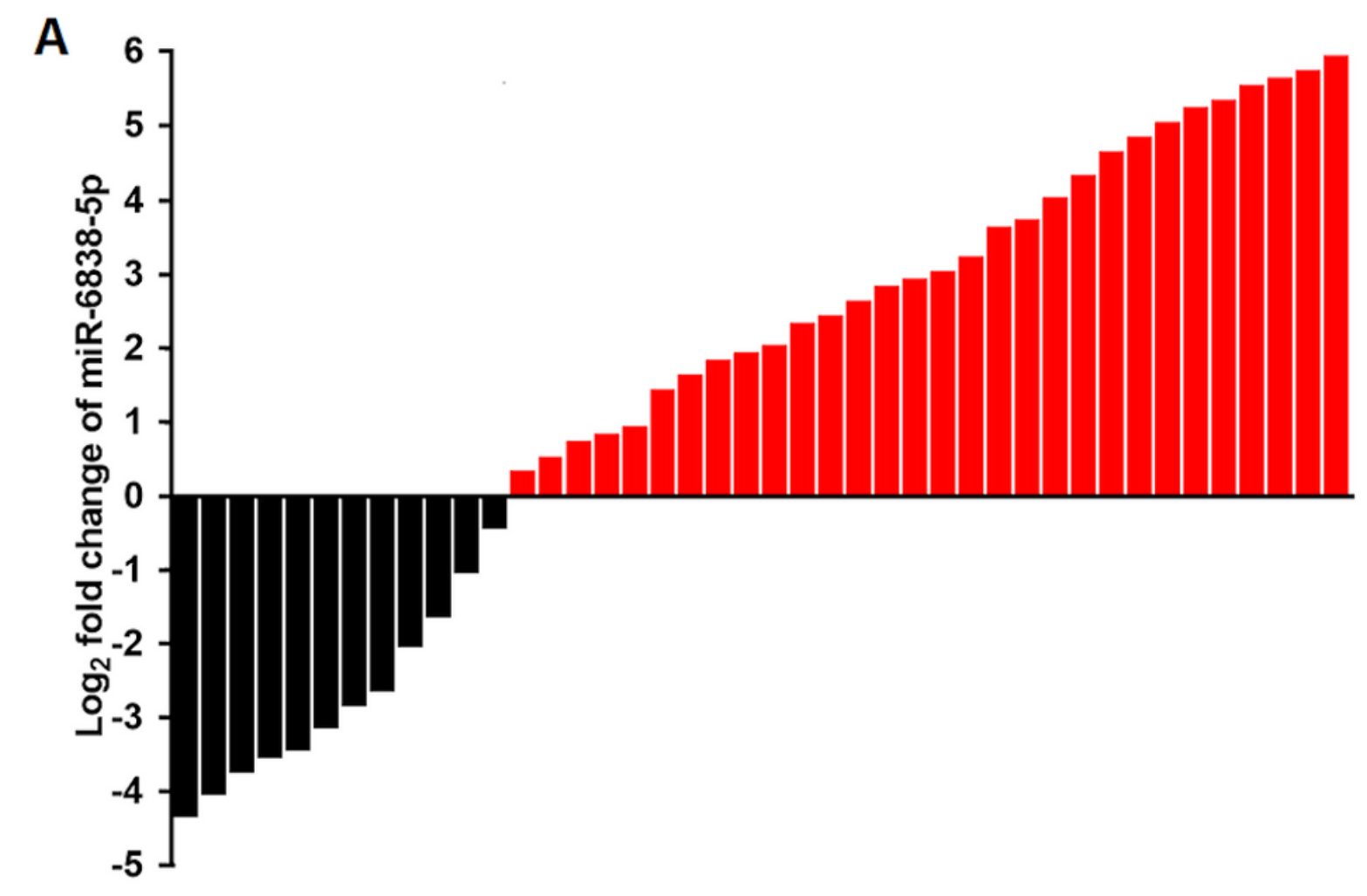

B

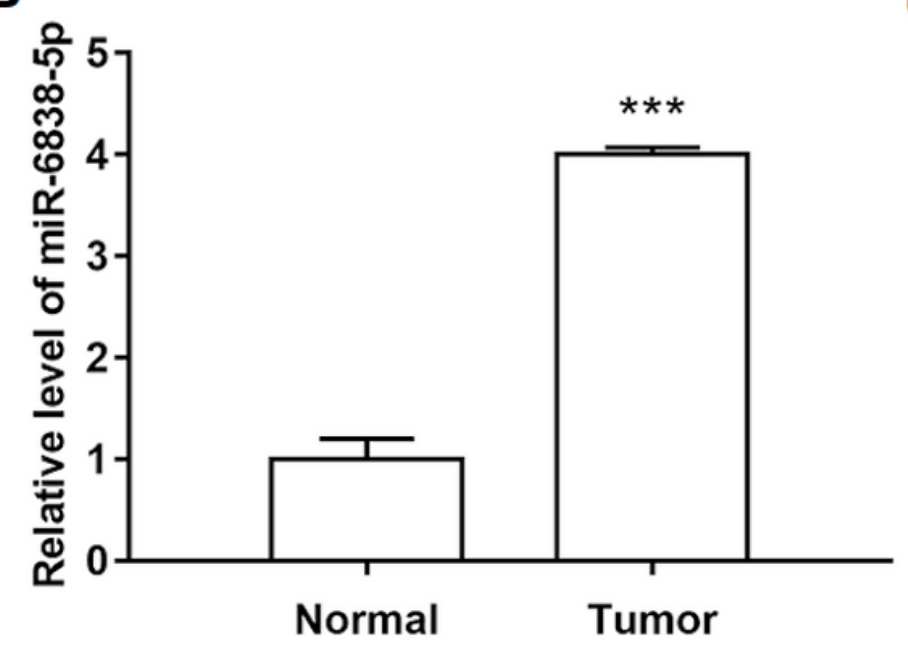

C

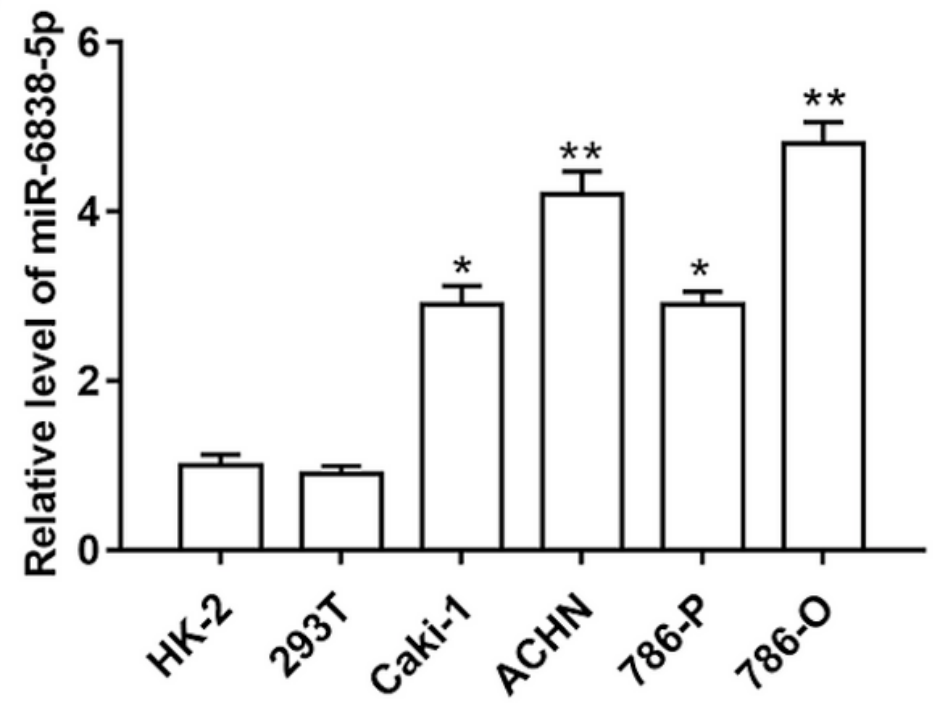

Figure 1

The relative level of miR-6838-5p was upregulated in RCC tissues and cells line. (A) Fold change of miR6838-5p in RCC tissues was elevated compared with adjacent normal tissues from each sample $(n=42)$.

(B) The relative expression of miR-6838-5p in RCC tissues was upregulated compared with normal tissues from all samples. ${ }^{\star \star \star} P<0.005$ versus normal. (C) Higher expression of miR-6838-5p was presented in RCC cell lines compared with normal RCC epithelial cell lines. * $P<0.05$ versus HK-2 or 293T, ** $\mathrm{P}<0.01$ versus HK-2 or 293T. 

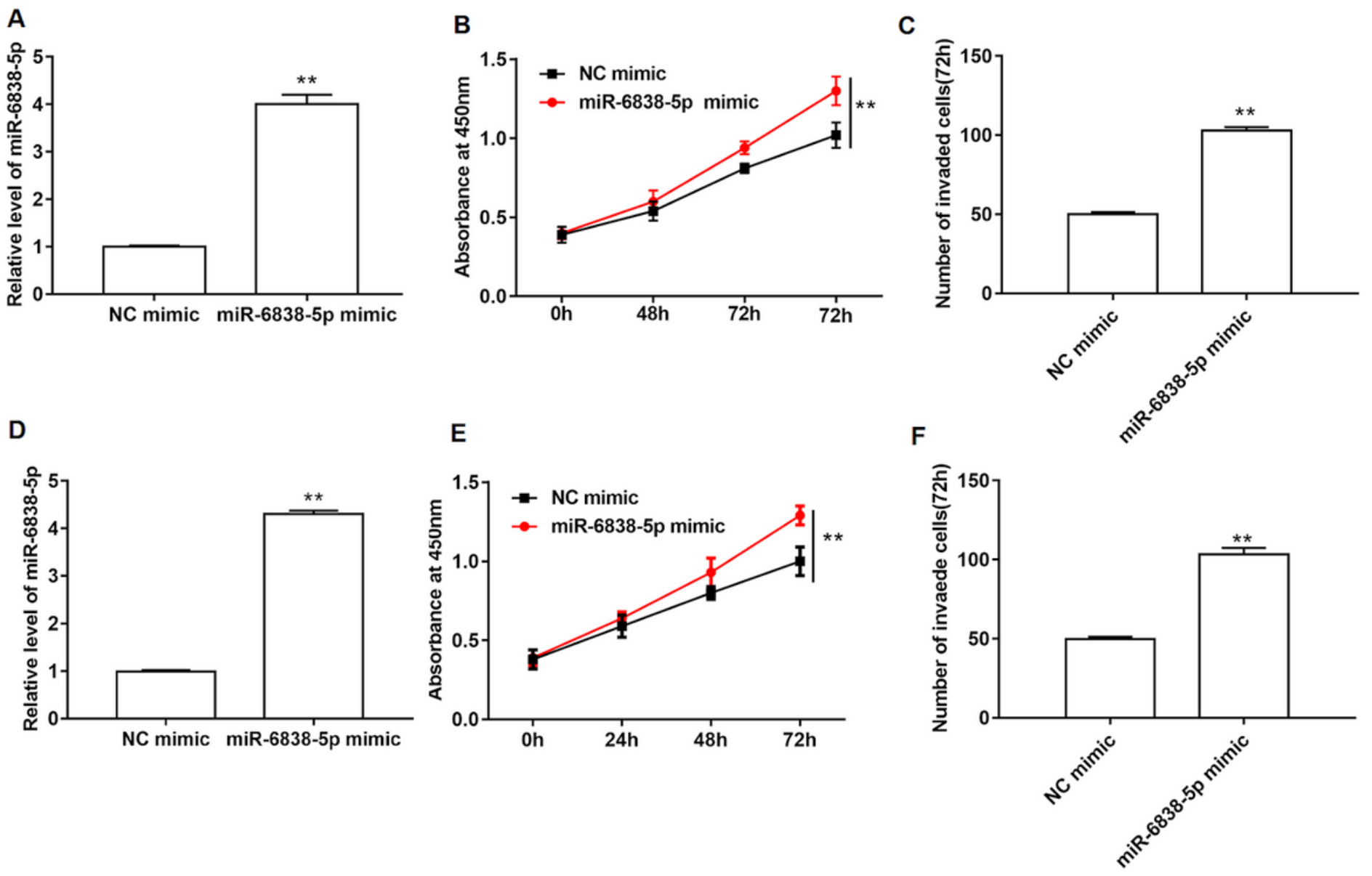

\section{Figure 2}

Overexpression of miR-6838-5p enhanced proliferation and invasion of ACHN and 786-0 RCC cells line. ACHN and 786-O RCC cell lines were transfected with NC mimic or miR-6838-5p mimic for $24 \mathrm{~h}$ and then the relevant indicators were detected. (A and D) The expression levels of miR-6838-5p in ACHN and 786-O RCC cell lines transfected with these two vectors were detected using RT-qPCR. ** $P<0.01$ versus NC mimic. (B and E) CCK-8 assay was used to assess the proliferative ability of ACHN and 786-0 cells line transfected with these two vectors for $0 \mathrm{~h}, 24 \mathrm{~h}, 48 \mathrm{~h}$ and $72 \mathrm{~h}$. ${ }^{*} \mathrm{P}<0.01$ versus NC mimic. ( $\mathrm{C}$ and $\mathrm{F}$ ) Transwell assay was used to assess the invasive ability of ACHN and 786-0 cells line transfected with these two vectors for $72 \mathrm{~h}$. 
A

D
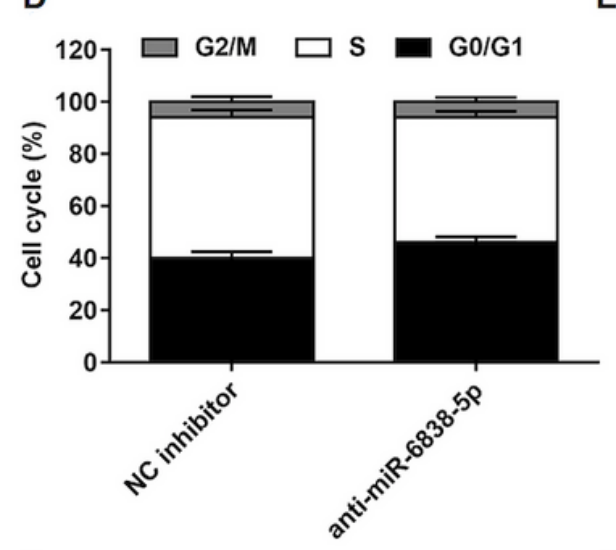

G

E
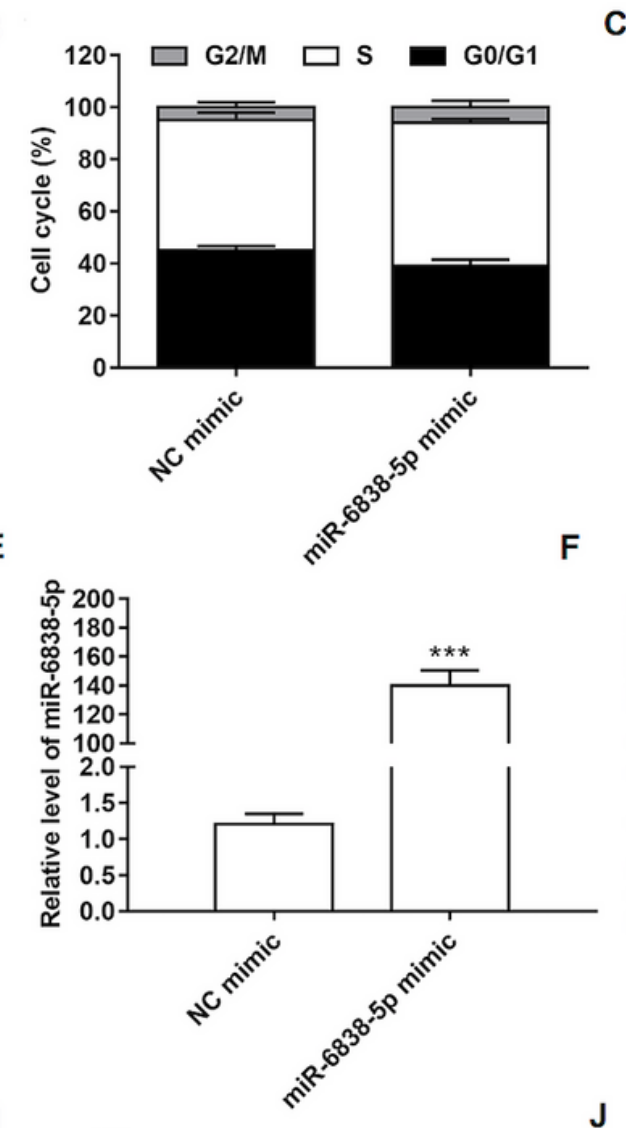

I

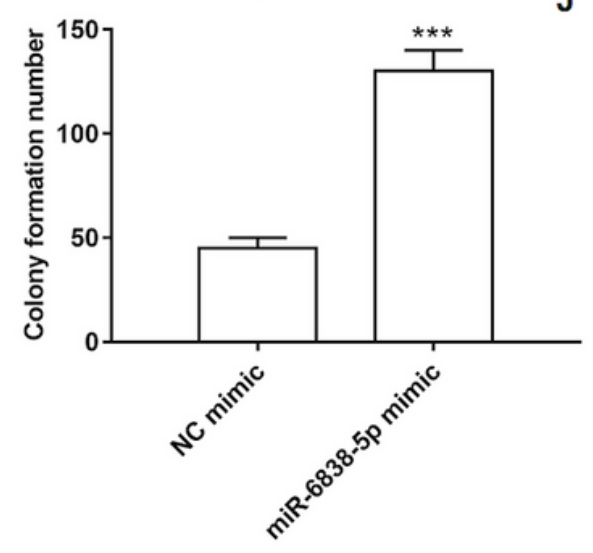

C

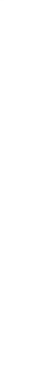

F
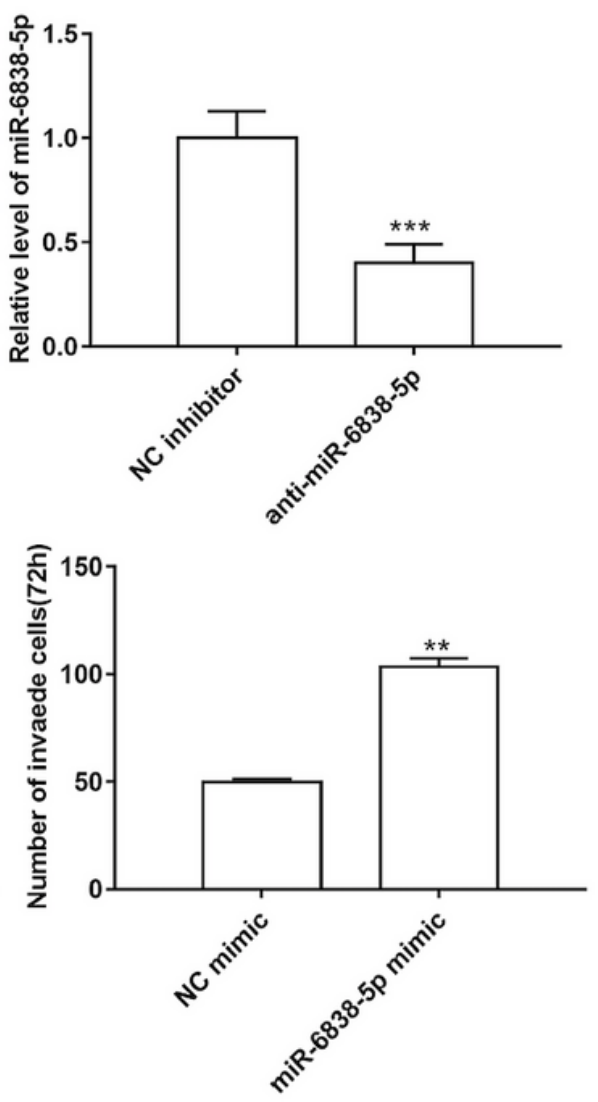

\section{Figure 3}

Effects of overexpression or inhibition of miR-6838-5p on cell cycle and colony formation. ACHN cells were transfected with NC mimic, miR-6838-5p mimic, NC inhibitor and anti-miR-6838-5p for $24 \mathrm{~h}$ and then the relevant indicators were detected. ( $A$ and $C$ ) Cell cycle of $A C H N$ cells transfected with these four vectors was determined by flow cytometry. (B and D) Quantify A-graph results with histogram. (C and F) The effects of these four vectors on the expression of miR-6838-5p were determined by RT-qPCR. $(G),(H)$, $(\mathrm{I})$ and $(\mathrm{J})$ The effect of ACHN cells transfected with these four vectors on colony formation. $* \star \star P<0.005$ versus NC mimic or NC inhibitor. 
A

WT DFTF1 3'UTR 5' GCCACACACAUUGUUGCUGCUA 3 '

$::::|||||| \mid$

miR-6838-5p : 3' UCCUCAGAACGGUGACGACGAA 5'

MUT DFTF1 3'UTR $5^{*}$ GCCACACACAUUGUGGACGCGA $3^{\prime}$

B

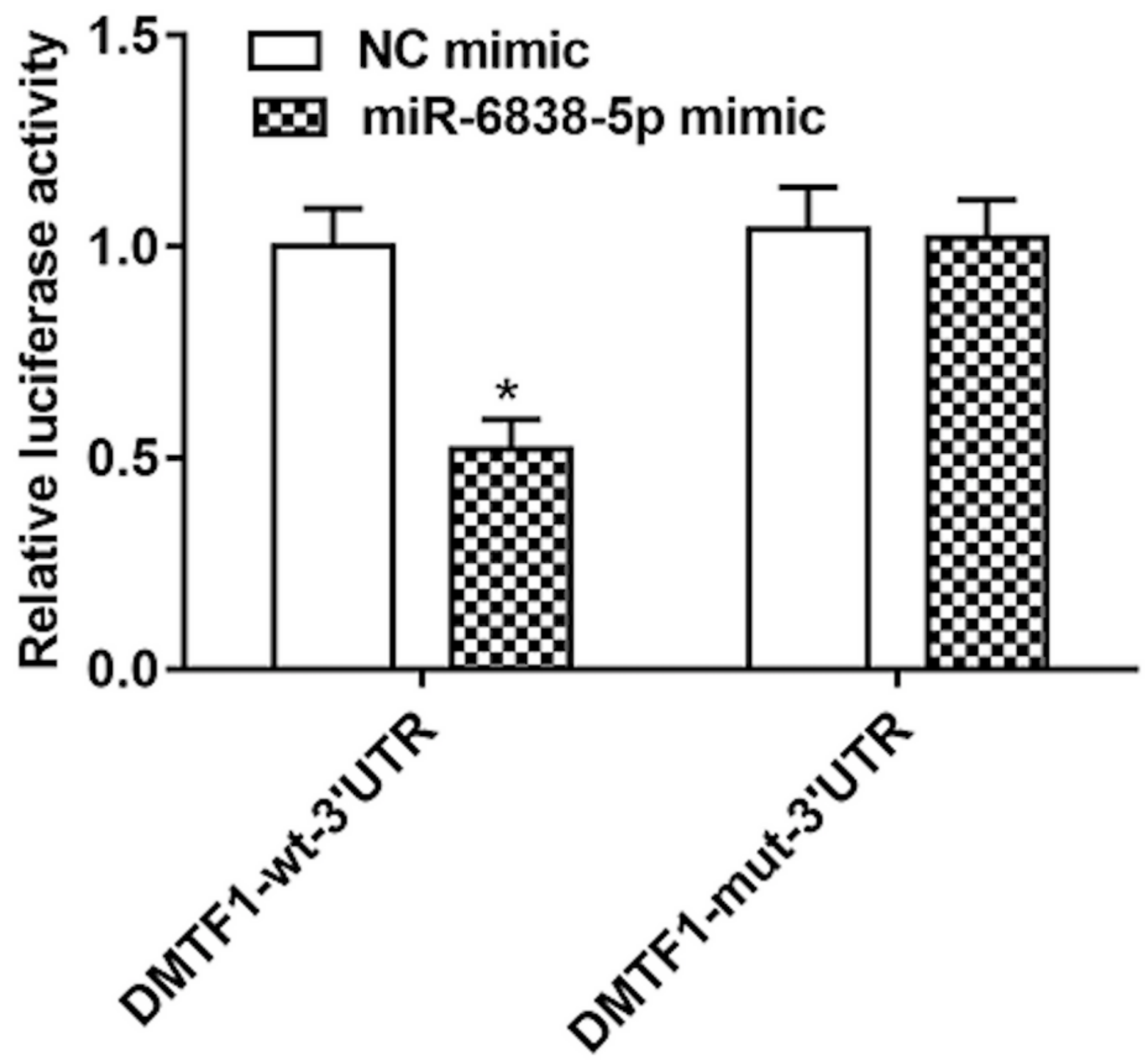

Figure 4

miR-6838-5p directly regulates the expression of DMTF1 via its 3'UTR. (A) Predicted results from the online resource TargetScan indicated a potential binding site of miR-6838-5p at the 3'UTR of DMTF1. (B) Luciferase activity assay showed a negative regulatory effect of miR-6838-5p on DMTF1 through the 3'UTR region. 


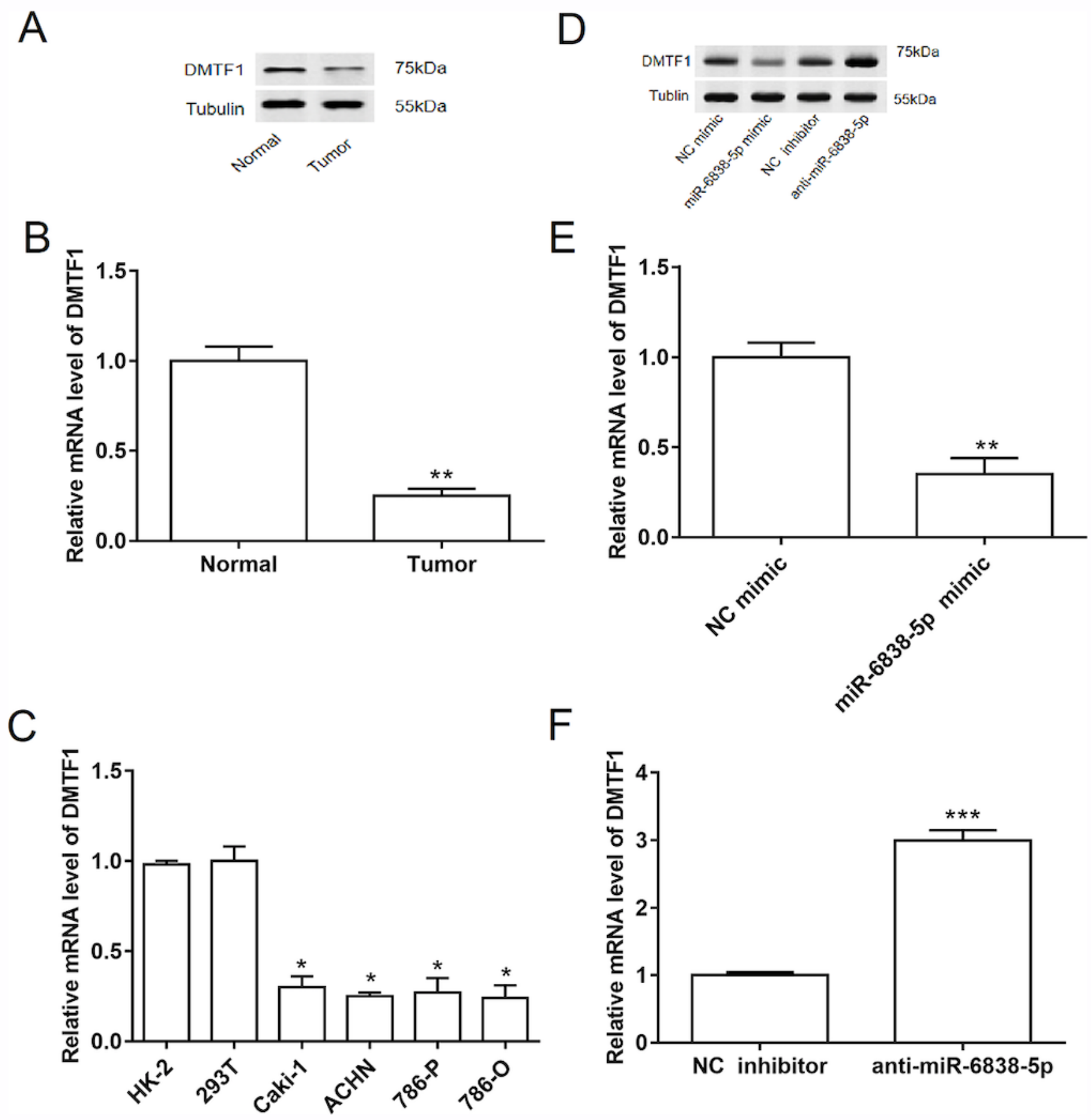

Figure 5

Expression of DMTF1 in RCC tissues and cell lines and effect of miR-6838-5p on DMTF1. Four vectors in Figure 4 were also used in Figure 5 to transfect ACHN cells. (A) Detection of DMTF1 mRNA in normal tissues and renal cell carcinoma tissues by RT-qPCR. (B) The histogram is the quantification of the A graph. ** $\mathrm{P}<0.01$ versus normal tissues. (C) The relative mRNA of DMTF1 was determined by RT-qPCR. * $\mathrm{P}<0.05$ versus HK-2 or 293T. (D) Expression of DMTF1 was evaluated by Western blotting while ACHN cells transfected with miR-6838-5p mimic or anti-miR-6838-5p. (E) The histogram is the quantification of the $D$ graph. ${ }^{* *} P<0.01$ versus NC mimic. ${ }^{* * *} P<0.005$ versus NC inhibitor. 
A

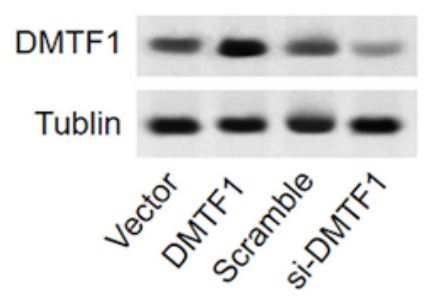

$75 \mathrm{kDa}$

$55 k D a$

B

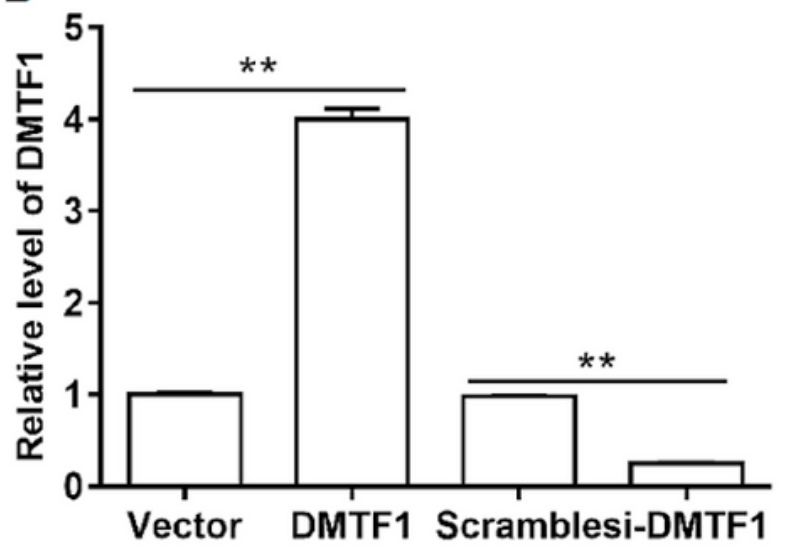

$\mathbf{E}$

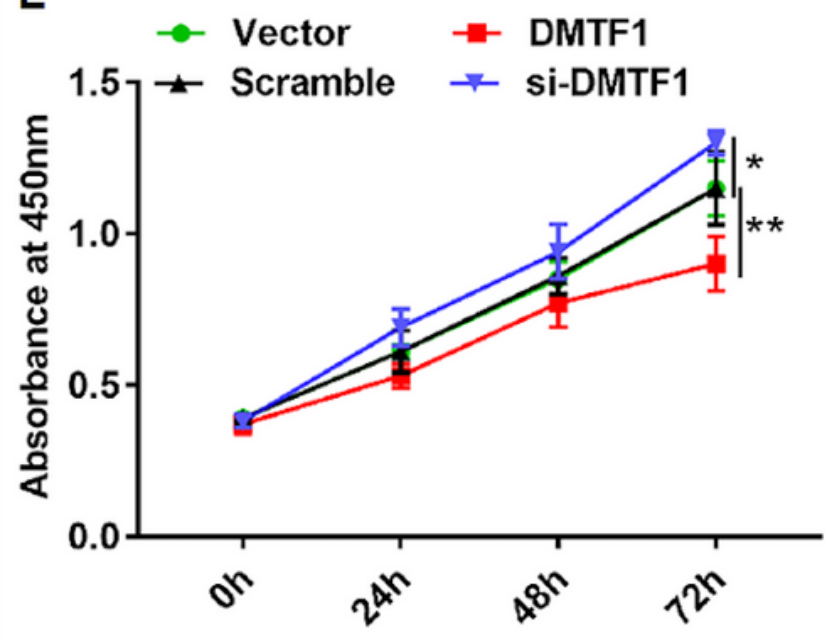

C

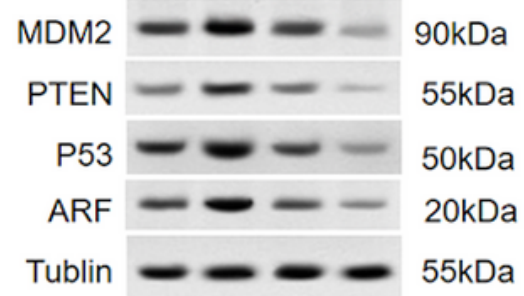

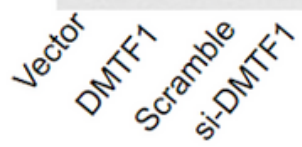

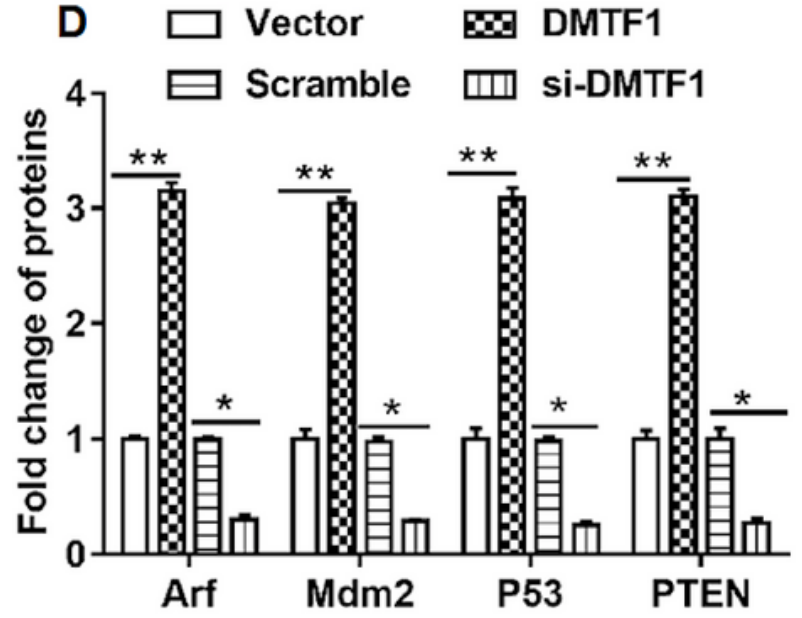

$\mathbf{F}$

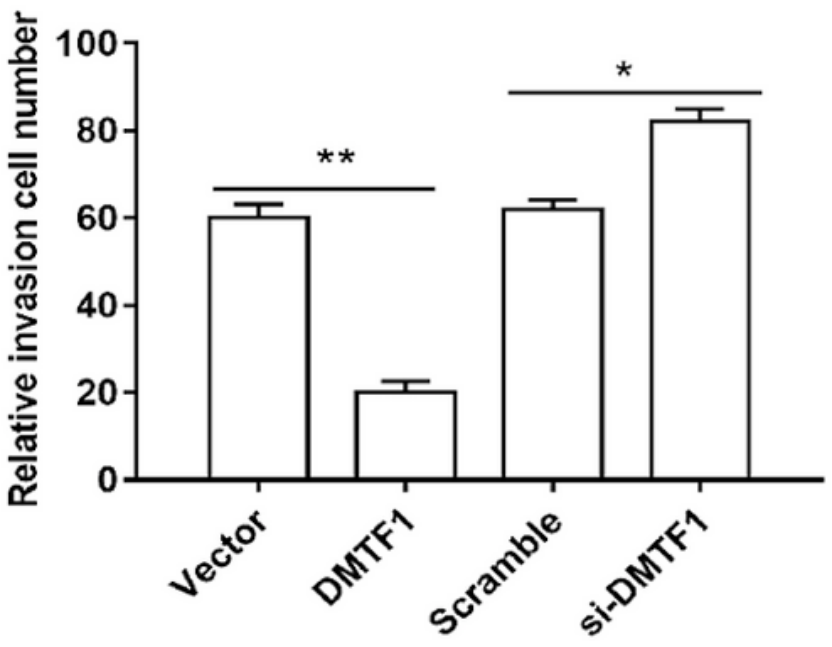

Figure 6

Overexpression or knockdown of DMTF1 on the downstream pathway of ARF-p53. Individual Vector, overexpression of DMTF1, Scramble or knockdown of DMTF1 transfected ACHN cells. (A) Effect of these four vectors on the expression of DMTF1 protein was detected by Western blotting. (B) The histogram is the quantification of the A graph. ${ }^{* *} \mathrm{P}<0.01$ versus vector or scramble. (C) Western blotting was used to detect the effect of these four vectors on the expression of ARF, MDM2, p53 and PTEN in ARF-p53 
pathway. (D) The histogram is the quantification of the $C$ graph. $* * P<0.01$ versus vector. ${ }^{*} P<0.05$ versus scramble. (E) CCK-8 assay was used to detect the effects of these four vectors on cell invasion for $0 \mathrm{~h}, 24 \mathrm{~h}, 48 \mathrm{~h}$, and $72 \mathrm{~h}$ after infecting ACHN cells. ${ }^{*} \mathrm{P}<0.05$ versus vector. ${ }^{*} \mathrm{P}<0.01$ versus scramble. (F) Transwell method was used to detect the effect of overexpression or knockdown of DMTF1 on cell invasion for $72 h . * P<0.05$ versus vector. ${ }^{*} P<0.01$ versus scramble.

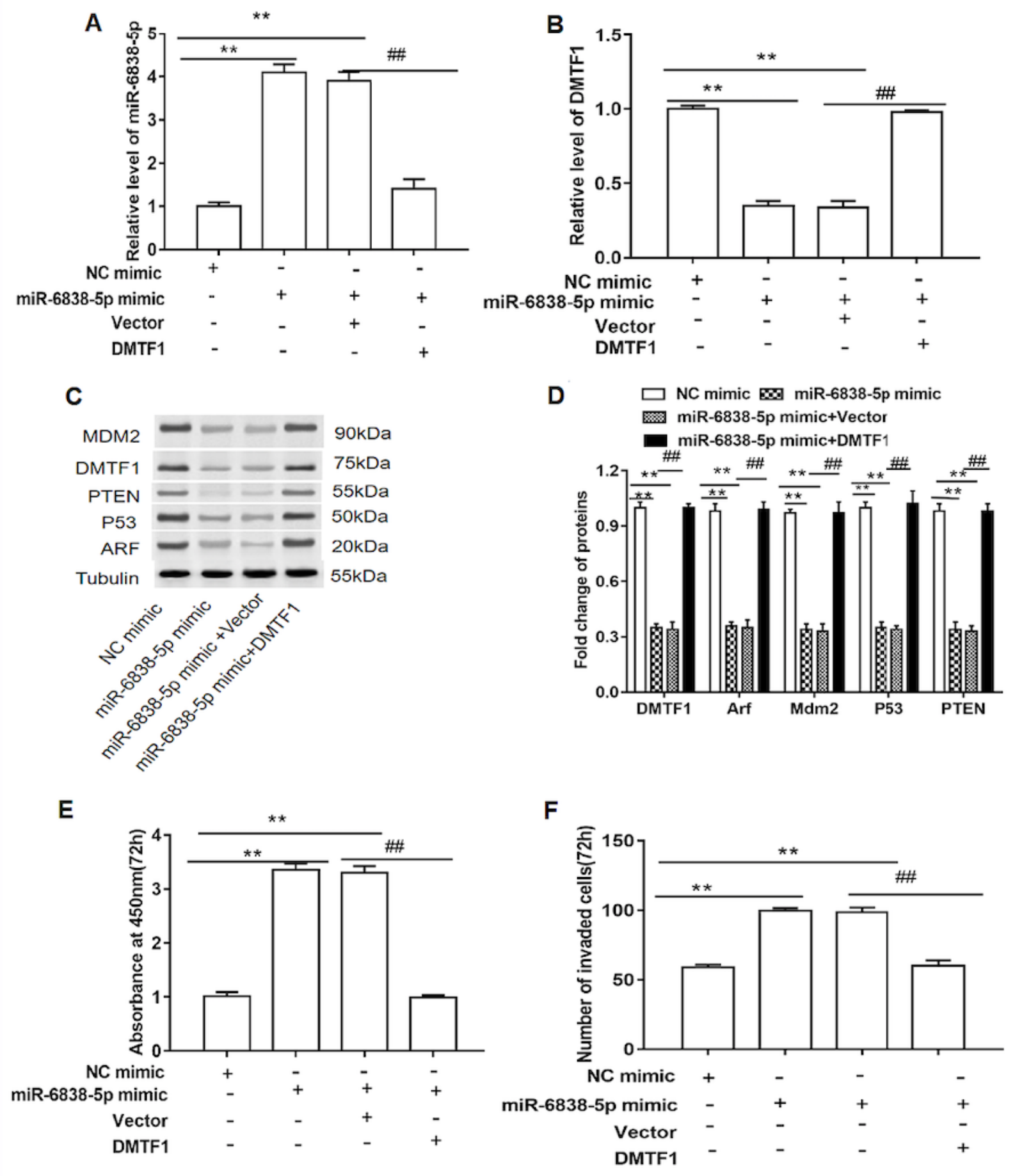

Figure 7 
Effect of overexpression of miR-6838-5p on the downstream pathway of DMTF1-p53. Individual NC mimic, miR-6838-5p mimic, or co-transfection of miR-6838-5p mimic and Vector, or co-transfection of miR-6838-5p mimic and overexpression of DMTF1 transfected ACHN cells. (A and B) The effects of the above four vectors on miR-6838-5p or DMTF1 expression were measured by RT-qPCR. ${ }^{*} P<0.01$ versus NC mimic. \#\#P $<0.01$ versus miR-6838-5p mimic and vector. (C-D) Western blot assay was used to detect the effects of the above four vectors on the expression of PTEN, p53, Mdm2, ARF, and DMTF1 after infecting ACHN cells. ${ }^{* *} \mathrm{P}<0.01$ versus NC mimic. \#\#P $<0.01$ versus miR-6838-5p mimic and vector. ( $E$ and F) The proliferation and invasiveness are determined by CCK-8 and Transwell assay of the above four vectors after infecting ACHN cells. ** $\mathrm{P}<0.01$ versus NC mimic. \#\#P $<0.01$ versus miR-6838-5p mimic and vector. 